\title{
AVALIAÇÃO DA CULTURA DE SEGURANÇA DO PACIENTE NO SETOR DE HEMODINÂMICA EM UM HOSPITAL PRIVADO DE SÃO LUIS - MA
}

\section{ARTIGO ORIGINAL}

MORAES, Inaldo Kley do Nascimento ${ }^{1}$

OLIVERIA, Sara Coelho ${ }^{2}$

DIBAI, Daniela Bassi ${ }^{3}$

DIAS, Rosane da Silva ${ }^{4}$

MORAES, Inaldo Kley do Nascimento. Et al. Avaliação da cultura de segurança do paciente no setor de hemodinâmica em um hospital privado de São Luis - MA. Revista Científica Multidisciplinar Núcleo do Conhecimento. Ano 05, Ed. 07, Vol. 02, pp. 43-56. Julho de 2020. ISSN: 2448-0959, Link de acesso: https://www.nucleodoconhecimento.com.br/saude/cultura-de-seguranca

\section{RESUMO}

Entende-se como segurança do paciente a redução ao mínimo aceitável dos riscos de danos associados ao cuidado de saúde. Esta depende de uma cultura de segurança formado a partir de um serviço qualificado e seguro, que envolva todos os profissionais do hospital, e de constante monitoramento dos fatores determinantes para o cuidado em saúde de qualidade. O objetivo geral do estudo foi avaliar a cultura

1 Mestrando em Gestão de Programas e Serviços de Saúde, especialista em Enfermagem em Cardiologia e hemodinâmica, Enfermeiro.

2 Estudante de Fisioterapia.

${ }^{3}$ Doutorado em Fisioterapia, Mestrado em Fisioterapia, graduada em fisioterapia.

${ }^{4}$ Orientadora. Doutorado em Ciências da Saúde, Especialização em traumatologia e ortopedia, graduada em fisioterapia. 
de segurança do paciente no setor de hemodinâmica em um hospital privado de São Luís. Trata-se de um estudo transversal, descritivo, quantitativo, realizado no setor hemodinâmico do centro de cardiologia do Hospital São Domingos, através do questionário HSOPSC - Hospital Survey on Patiente Safety Culture dividido em 12 dimensões a percepção sobre cada uma deles se descreve pela porcentagem de respostas positivas. Participaram 69 profissionais, destes a maioria $47(68 \%)$ eram mulheres, 16(23\%) eram de enfermeiros; 30 (43\%) trabalhavam no hospital, entre 1 a 5 anos $33(48 \%)$ com carga horária semanal entre 20 a 39 horas. No tocante às dimensões avaliadas a "Expectativas sobre o supervisor/chefe" (79\%); "Aprendizado organizacional / melhoria continuada" (90\%); "Trabalho em equipe dentro da unidade" com $(78 \%)$ e "apoio da gestão hospitalar" $(91 \%)$ foram consideradas fortes. Além disso, 52(68\%) dos participantes relataram não ter enviado ou preenchido nenhum relatório de evento adverso nos últimos 12 meses. Portanto, a cultura de segurança do paciente do setor tem potencial de melhora em quase todas as dimensões avaliadas.

Palavras-chave: Eventos adversos, gestão de riscos, segurança do paciente, cultura de segurança do paciente, assistência.

\section{INTRODUÇÃO}

Nos últimos anos a importância de cultura de segurança eficaz vem ganhando cada vez mais atenção do campo das organizações de saúde, por ser essencial no âmbito hospitalar na tentativa de melhorar os cuidados de saúde, e assim, reduzir os riscos de eventos adversos. Uma vez que, devido o aumentando de complexidade dos cuidados de saúde, as chances de ocorrência de incidentes, erros e falhas se tornaram maiores (REIS, 2013).

É importante ressalta que, entende-se como segurança do paciente a redução ao mínimo aceitável dos riscos de danos associados ao cuidado de saúde (BRASIL, 2013). Esta depende de uma cultura de segurança formado a partir de um serviço qualificado e seguro, definido a partir do comportamento em equipe e individual 
independentemente da posição hierárquica ocupada (ALVES et. al., 2015; OLIVEIRA et. al., 2014).

No intuito de melhorar a cultura de segurança do paciente no Brasil, o Ministério da saúde no ano de 2013 criou a Instituição do Programa Nacional de Segurança do Paciente (PNSP), como objetivo a prevenir, monitorar e reduzir os danos e erros causados aos pacientes durante o cuidado de saúde através da implementação da gestão de risco e dos Núcleos de Segurança do Paciente nas diversas áreas de atenção, gestão e organização de serviços de saúde (BRASIL, 2013).

Durante os primeiros 3 anos após a criação do PNSP, foram implantados um total de 2.333 Núcleos de Segurança do Paciente (NSP) no país. No mesmo período foi possível observar um aumento expressivo do número de notificações de eventos adversos (BRASIL, 2016). Durante o período de janeiro de 2014 a julho de 2017, foi notificado à ANVISA um total de 50.735 incidentes relacionados à assistência à saúde, sendo que nos serviços de hemodiálise 224 incidentes foram notificados (BRASIL, 2017).

Entre os danos ocasionados ao paciente em decorrência de falha a assistência do cuidado de saúde, destaca-se o aumento do tempo de internação, dos custos hospitalares, necessidade de novas interversões, custos sociais, danos emocionais e, em caso mais graves, óbito (SIMAN; CUNHA; BRITO, 2017; MILAGRES, 2015). Além disso, os profissionais de saúde envolvidos devido à falta de compreensão e o caráter punitivo ainda existente em algumas instituições podem vir a se sentir culpados, com medo, vergonha e constrangidos. Fato que contribui para que estes não notifiquem o evento adverso ocorrido (DUARTE et. al., 2015; DIAS et al., 2014).

Em estudo realizado por Neves (2017), com o objetivo de avaliar as hemorragias em doentes submetidos a um cateterismo cardíaco, verificou que dos 616 casos incluídos no estudo, em 95 houve eventos hemorrágicos (15,4\%) até 1 hora após o cateterismo na primeira avaliação e 343 eventos hemorrágicos (55,7\%) até 48 horas durante a segunda avaliação; além disso, 9 (1,5\%) óbitos foram registrados até a primeira hora 
após o cateterismo e $12(1,9 \%)$, até 48 horas após cateterismo. O que evidencia um número alto de eventos hemorrágicos.

Embora as instituições estejam compromissadas em dar melhor assistência ao paciente e reduzir os eventos adversos através de medidas e protocolos de segurança que garantam tal efeito. Setores que realizam procedimentos invasivos, como o setor de hemodinâmica, não possuem protocolos bem definidos o contrário do que se observam nos setores de urgência e emergência, UTI e CDI que possuem protocolos já estabelecidos.

Desta forma, considerando a importância que a discussão do tema tem para uma assistência mais segura do paciente e visando contribuir para a redução de riscos e dos impactos negativos que os eventos adversos acarretam em diferentes aspectos na vida do paciente e da dos profissionais da área de saúde, o presente estudo teve como finalidade avaliar a cultura de segurança do paciente no setor de hemodinâmica em um hospital privado de São Luís (MA).

\section{METODOLOGIA}

Trata-se de um estudo transversal, de natureza descritiva com abordagem quantitativa, realizado no setor de hemodinâmica do hospital São Domingos em São Luis- MA. Sendo este considerado um hospital de alta complexidade, devido à tecnologia empregada em procedimentos e os serviços prestados em todas as áreas da medicina, realizando cirurgias de grande porte, vascular, urológicas, oncológicas, emergências clínicas, cirúrgicas, cardiológicas, cirurgias cardíaca, nefrologia, neurologia, traumatologia.

A amostragem foi não probabilística, deu-se por conveniência, considerando o quadro de profissionais do setor. Utilizou-se como critério de inclusão estar trabalhando no setor independente do tempo de serviço e como critério de exclusão estar afastado por licença médica, em período de férias e os questionários preenchidos de maneira errada ao ponto de prejudicar a análise das variáveis. Oito questionários foram 
desprezados por cumprirem o último critério de exclusão, resultando em uma amostra de 69 profissionais.

Para avaliação a cultura de segurança dos profissionais utilizou-se o HSOPSCHospital Survey on Patiente Safety Culture, criado pela Agency for Health Care Research And Quality (AHRQ) e validado no Brasil por Reis (2013). O HSOPSC compõe-se de questionamentos sobre a segurança do paciente, eventos adversos, liderança, comunicação e gestão, possibilita conhecer a percepção sobre a unidade de trabalho, e de forma geral sobre toda a instituição, dá ênfase aos erros, às percepções de erros e às dimensões da cultura de segurança do paciente (REIS, 2013).

É composto por 42 questões divididas em 12 dimensões, sendo elas: Frequência de eventos notificados; Percepção de segurança; Expectativas sobre supervisor/ chefe; Aprendizagem organizacional / melhoria continuada; Trabalho em equipe na unidade; Abertura da comunicação; retorno da informação e comunicação sobre erros; Resposta não punitiva para erros; adequação de profissionais; Apoio da gestão hospitalar; Trabalho em equipe entre as unidades e passagem de plantões/ transferências. Além das 12 dimensões, o HOSPSC possui questões a respeito do número de notificações de incidentes de segurança, nota geral da segurança do paciente e aspectos laborais dos profissionais.

As possibilidade de respostas para cada questão foi baseada no tipo likert de 5 alternativas: ( $1=$ discordo fortemente, $2=$ discordo levemente, $3=$ nem discordo nem concordo ou às vezes, $4=$ concordo levemente e $5=$ concordo fortemente). Para a interpretação dos dados, os escores de cada item foram convertidos em uma pontuação que totaliza 100 pontos, considerado o desejável. Dessa forma, os escores passaram a assumir os seguintes valores: discordo fortemente $=0$, discordo levemente $=25$, neutro $=50$, concordo levemente $=75$ e concordo fortemente $=100$. Alguns itens apresentam escore reverso, ou seja, nestas questões um escore reduzido representa uma atitude positiva. 
O percentual de respostas positivas para cada dimensão foi obtido através da seguinte fórmula (SORRA; NIEVA, 2004, p. 35): "\% de respostas positivas da dimensão $\mathrm{X}=$ [número de respostas positivas aos itens da dimensão $\mathrm{X} /$ número total de respostas válidas aos itens da dimensão $X$ (positivas, neutras e negativas, excluindo-se os dados ausentes)] x 100"

O percentual de respostas positivas permite identificar áreas fortes e frágeis na segurança do paciente. Foram consideradas "áreas fortes da segurança do paciente" no hospital as dimensões que obtiveram mais de $75 \%$ de respostas positivas. De modo semelhante, "área frágeis da segurança do paciente" e que necessitam melhorias as que obtiveram $50 \%$ ou menos de respostas positivas (REIS, 2013).

Os dados foram avaliados pelo programa IBM SPSS Statistics 20 (2011). Os dados descritivos das variáveis categóricas foram apresentados por frequência absoluta e percentuais. Depois foi feita a estimativa da média e do intervalo de confiança de $95 \%$ (IC 95\%) da porcentagem de respostas positivas de cada uma das 12 dimensões da cultura de segurança.

Após concordar em participar da pesquisa, cada participante recebeu uma cópia do instrumento e duas cópias do Termo de Consentimento Livre e Esclarecido (TCLE). Após assinatura do TCLE pelo participante, o mesmo recebeu uma via e a outra ficou de posse do pesquisador. Esta pesquisa foi aprovada pelo Comitê de Ética em Pesquisa com Seres Humanos da Universidade CEUMA Sob o parecer 3.107.901.

\section{RESULTADOS}

Participaram do estudo 69 profissionais. Destes 47 (68\%) eram do sexo feminino, quanto a faixa etária houve predomínio de indivíduos com idade entre 31 a 40 anos. Dentre os profissionais que responderam à pesquisa 15 (22\%) eram médicos, 16(23\%) enfermeiros, 15(22\%) técnico de enfermagem, 9 (13\%) técnicos em radiologia, 9(13\%) auxiliar administrativo, 4(6\%) hotelaria e 1(1\%) psicólogo ( Tabela 1). 
Tabela 1. Dados sociodemográfico dos profissionais participantes do estudo, Maranhão, Brasil, 2019 ( $\mathrm{N}=69)$.

\begin{tabular}{|l|l|l|}
\hline Variáveis & N & $\%$ \\
\hline Sexo & & \\
\hline Feminino & 47 & 68 \\
\hline Masculino & 22 & 32 \\
\hline Idade & & \\
\hline De 19 a 30 anos & 27 & 39 \\
\hline De 31 a 40 anos & 28 & 41 \\
\hline De 41 a 50 anos & 12 & 17 \\
\hline De 51 a $\mathbf{6 4}$ anos & 2 & 3 \\
\hline Profissão & & \\
\hline Médico & 15 & 22 \\
\hline Enfermeiro & 16 & 23 \\
\hline Técnico de enfermagem & 15 & 22 \\
\hline Técnico em radiologia & 9 & 13 \\
\hline Auxiliar administrativo & 9 & 13 \\
\hline Hotelaria & 4 & 6 \\
\hline Psicólogo & 1 & 1 \\
\hline Total & 69 & 100 \\
\hline
\end{tabular}

Fonte: Elaborada pelo autor (2019).

As variáveis tempo de trabalho na especialidade, tempo de trabalho no hospital e tempo de trabalho na unidade houve predomínio de respostas para o período de 1 a 5 anos com respectivos percentuais de $28(41 \%) ; 30(43 \%)$ e 36(52\%). Quanto à carga horária semanal, 33 (48\%) relataram entre 20 a 39 horas de trabalho (Tabela 2). 
Tabela 2. Características profissionais dos participantes do estudo, Maranhão, Brasil, 2019(N=69).

\begin{tabular}{|c|c|c|}
\hline Variáveis & $\mathrm{N}$ & $\%$ \\
\hline \multicolumn{3}{|l|}{ Contato direto com paciente } \\
\hline Sim & 60 & 87 \\
\hline Não & 9 & 13 \\
\hline \multicolumn{3}{|c|}{$\begin{array}{l}\text { Tempo de trabalho na especialidade/ profissão } \\
\text { atual }\end{array}$} \\
\hline Menos de 1 ano & 7 & 10 \\
\hline De 1 a 5 anos & 28 & 41 \\
\hline De 6 a 10 ano & 22 & 32 \\
\hline De 11 a 15 anos & 7 & 10 \\
\hline De 21 ou mais anos & 5 & 7 \\
\hline \multicolumn{3}{|c|}{ Tempo de trabalho neste hospital } \\
\hline Menos de 1 ano & 6 & 9 \\
\hline De 1 a 5 anos & 30 & 43 \\
\hline De 6 a 10 ano & 24 & 35 \\
\hline De 11 a 15 anos & 4 & 6 \\
\hline De 16 a 21 anos ou mais & 5 & 7 \\
\hline \multicolumn{3}{|c|}{ Tempo de trabalho na área/unidade atual } \\
\hline Menos de 1 ano & 12 & 17 \\
\hline De 1 a 5 anos & 36 & 52 \\
\hline De 6 a 10 ano & 22 & 32 \\
\hline De 11 a 15 anos & 3 & 4 \\
\hline De 16 a 21 anos ou mais & 4 & 6 \\
\hline \multicolumn{3}{|l|}{ Carga horária semanal } \\
\hline Menos de 20 horas & 8 & 12 \\
\hline Entre 20 a 39 horas & 33 & 48 \\
\hline Entre 40 a 59 horas & 21 & 30 \\
\hline Entre 60 a 79 horas & 3 & 4 \\
\hline
\end{tabular}


Entre 80 a 100 horas ou mais

Total
4

69
6

100

Fonte: Elaborada pelo autor (2019).

No tocante às dimensões avaliadas, quatro delas aparecem com avaliação de cultura fortalecida, por terem mais que $75 \%$ de respostas positivas, são elas: "Expectativas sobre o supervisor/ chefe" (79\%); "Aprendizado organizacional/ melhoria continuada" (90\%); "Trabalho em equipe dentro da unidade" (78\%) e "Apoio da gestão hospitalar" com (91\%). Em contrapartida, a dimensão "Resposta não punitiva" com (48\%) foi considerada área de fragilidade da segurança do paciente, por ter menos que $50 \%$ de respostas positivas (Tabela 3 ).

Tabela 3. Número de repostas positiva para cada dimensão do questionário HSOPSC e seus respectivos porcentuais, médias e IC 95\%, Maranhão, Brasil, 2019 (N=69).

\begin{tabular}{|l|l|l|l|l|}
\hline Dimensões & N & $\%$ & MÉDIA & IC 95\% \\
\hline D1 - Percepção geral de segurança & 172 & 62 & 43 & $39,91-46,09$ \\
\hline D2 - Frequência de relatos de eventos & 121 & 59 & 40,3 & $37,76-42,91$ \\
\hline D3 - Expectativas sobre o supervisor/ chefe & 217 & 79 & 54,25 & $50,78-57,72$ \\
\hline D4 - Aprendizado organizacional/ melhoria & 187 & 90 & 62,3 & $59,12-65,54$ \\
\hline continuada* & & & & \\
\hline D5 - Trabalho em equipe dentro da unidade & 214 & 78 & 53,5 & $50,06-56,94$ \\
\hline D6 - Abertura da comunicação & 105 & 51 & 35 & $32,62-37,38$ \\
\hline D7 - Respostas não punitiva+ & 99 & 48 & 33 & $30,66-35,34$ \\
\hline D8 - Adequação de profissionais & 178 & 65 & 44,5 & $41,36-47,64$ \\
\hline D9 - Retorno da informação e comunicação & 141 & 68 & 47 & $44,22-49,78$ \\
\hline D10 - Apoio da gestão hospitalar * & 188 & 91 & 62,6 & $59,46-65,88$ \\
\hline D11 - Trabalho em equipe entre as unidades & 201 & 73 & 50,25 & $46,92-53,58$ \\
\hline D12 - Passagem de plantões / transferências & 167 & 61 & 41,75 & $38,73-44,77$ \\
\hline
\end{tabular}


+ Dimensões que apresentam fragilidades (D7); * Dimensões que apresentam fortalezas (D3; D4; D5 e D10).

Fonte: Elaborada pelo autor (2019).

Quanto à notificação de evento adverso, 47 (68\%) dos participantes relataram não ter enviado ou preenchido nenhum relatório de evento adverso nos últimos 12 meses. $\mathrm{O}$ maior número de relatórios enviados entre os participantes foi entre 1 e 2 relatórios, $15(22 \%)$ dos participantes se encaixaram nesta categoria (Gráfico 1).

Gráfico 1. Número de relatórios de evento adverso preenchido e enviado nos últimos 12 meses, Maranhão, Brasil, 2019 ( $\mathrm{N}=69)$.

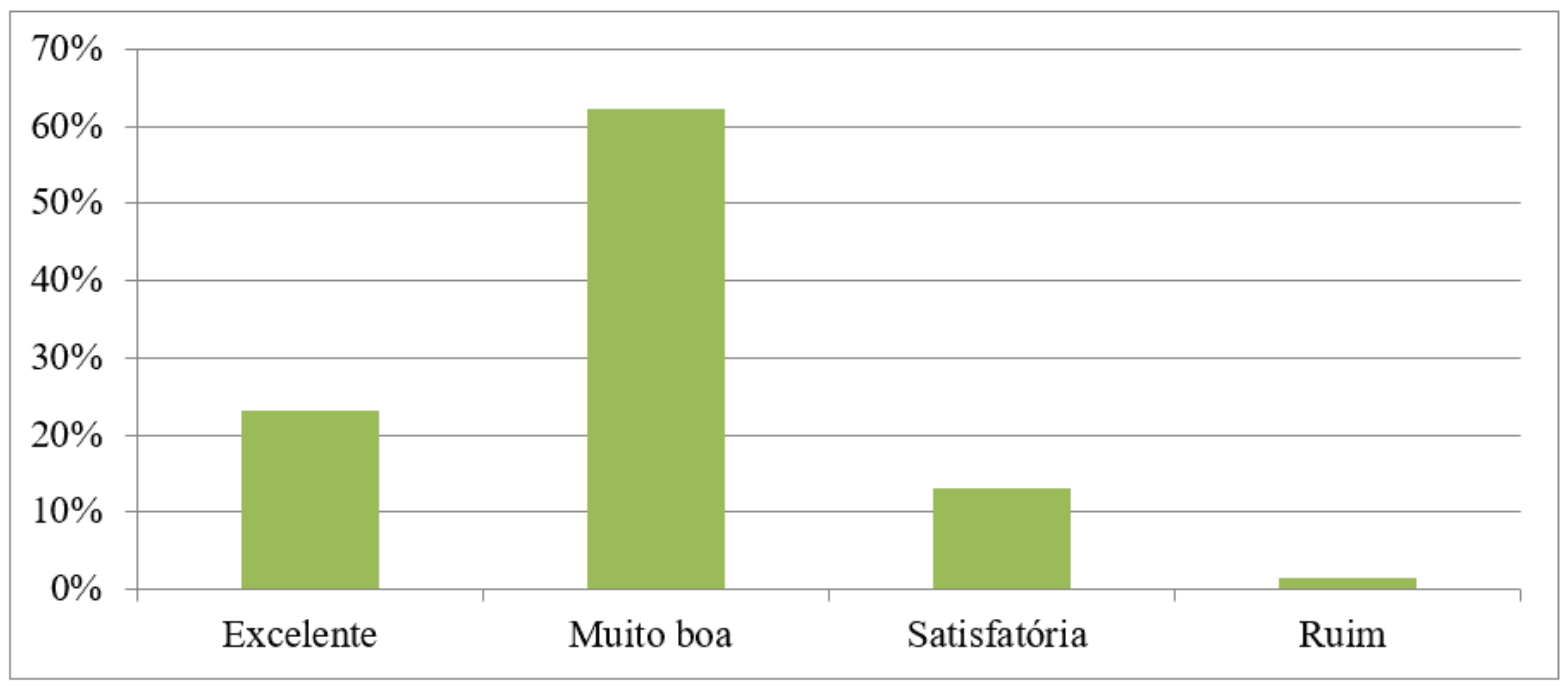

Fonte: Elaborada pelo autor (2019).

Quanto a avaliação do grau de segurança, 43 (62\%) dos participantes a consideraram muito boa, $16(23 \%)$ a consideraram excelente, $9(13 \%)$ avaliaram a segurança do setor como satisfatória e a minoria 1(1\%) avalia como ruim (Gráfico 2). 
Gráfico 2. Avaliação do grau de segurança do setor hemodinâmico, segundo os participantes, Maranhão, Brasil, 2019(N=69).

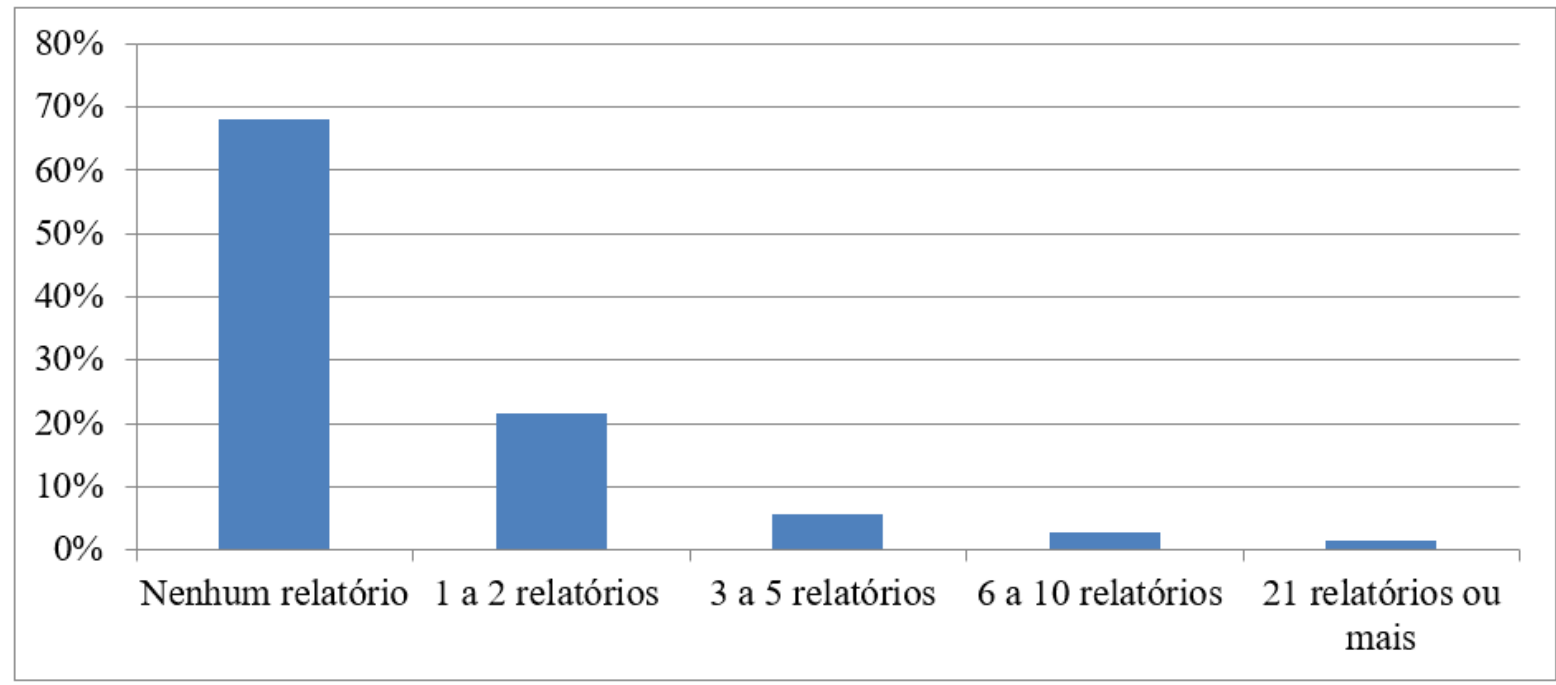

Fonte: Elaborada pelo autor (2019).

\section{DISCUSSÃO}

Os resultados do presente estudo evidenciaram potencial de melhora na cultura de segurança do paciente no setor de hemodinâmica em quase todas as dimensões avaliadas, uma vez que apenas 4 das 12 dimensões avaliadas foram classificadas como fortalecidas, por obterem mais de $75 \%$ de respostas positivas.

A dimensão "resposta não punitivas aos erros" também classificada como fraca nos estudos realizados por Andrade et al.(2018); Santiago e Turrini (2015) e Mello e Barbosa(2013) em que a dimensão recebeu (36,1\%), (18\%) e (18\%) de respostas positivas, respectivamente. Além disso, 32 (46\%) dos participantes do presente estudo responderam que "concordam" ou "concordam totalmente" com o item "os profissionais consideram que seus erros podem ser usados contra eles" o qual compõe a dimensão, o que sugere a presença de um caráter punitivo no setor.

A análise da seção que investigou o número de eventos adversos relatados nos últimos 12 meses pelos participantes afirma este pensamento, dado ao fato de que $47(68 \%)$ da equipe referem não ter relatado nenhum relatório nos últimos 12 meses. 
Resultados semelhantes foram encontrados em um estudo realizado em uma unidade de terapia intensiva em que foi aplicado para 59 profissionais de saúde o questionário Pesquisa sobre Segurança do Paciente em Hospitais, também usado no presente pesquisa, alerta para a subnotificações de eventos adversos em que a maioria dos profissionais não tinha realizado notificações durante o período estudado o que pode ser influenciado pela cultura punitiva que pode vir a influenciar bloqueia novas notificações e melhoras na segurança do paciente (MINUZZ; SALUM; LOCKS, 2016).

Além disso, embora, segundo os profissionais, eles relatem com frequência os eventos adversos ocorridos no setor em casos em que o erro é identificado e corrigido antes de afetar o paciente e quando ocorrem erros com potencial ou sem de prejudicar o paciente, os resultados encontrados na dimensão "Frequência de relatos de eventos" contradizem os encontrados na quantidade de notificações realizadas nos últimos 12 meses em que foi observado um baixo número de relatórios. Em estudo desenvolvido por Costa (2014), em dois hospitais públicos no Paraná, com 282 profissionais, encontrou resultados semelhantes em que $(77,8 \%)$ dos profissionais não tinham realizado notificação nos últimos 12 meses e segundo a dimensão "Frequência de relatos de eventos" os funcionários relatavam com frequência os erros existentes. Fato que demonstra a importância do incentivo à notificação e orientação de como realizá-la de maneira adequada para que a percepção dos funcionários e o número de relatórios realizados se equiparem.

O alto percentual de respostas positivas (90\%) a dimensão "Aprendizado organizacional/ melhoria continuada" e "Apoio da gestão hospitalar" (91\%) demonstra que os profissionais percebem a existência de medidas de melhoria continuada na instituição que visam aprimorá-los e capacitá-los (WEGNER et al., 2016). (86,9\%) dos profissionais responderam positivamente ao item "erros têm levado a mudanças positivas por aqui" que compõem a dimensão "Aprendizado organizacional/ melhoria continuada", demonstrando um olhar positivo sobre tal característica.

Embora não seja classificada como forte, a dimensão "Retorno da informação e comunicação" recebeu em percentual significativo de respostas positivas $(68,1 \%)$ se comparado aos encontrados na literatura, como nos estudos de Mello e Barbosa 
(2017) e Minuzzi et. al.(2016) que obtiveram respectivamente $(27,1 \%)$ e $(20,81 \%)$ de respostas positivas a esta dimensão, composta pelos itens "nós recebemos informações sobre mudanças implementadas a partir dos relatórios de eventos", "nós somos informados sobre os erros que acontecem nesta unidade" e "nesta unidade discutimos meios de prevenir erros evitando que eles aconteçam novamente". O que sugere que, mesmo com um possível déficit de notificação, as que são realizadas são usadas a fim de evitar a reincidências.

Outro ponto que diverge dos dados encontrados na literatura discerne acerca da avaliação de segurança do paciente e a dimensão "percepção geral de segurança", ambas com percentuais de (62\%) para muito boa. Já em estudos realizados por Mello e Barbosa (2017), em duas UTI's adulto de hospitais públicos, em que a resposta de (77,7\%) para o grau de segurança foi muito boa/aceitável, diverge do baixo percentual de respostas positivas da dimensão "percepção do grau de segurança" (25,9\%). Assim como Tomazoni et. al. (2015), em estudo descritivo-exploratório em quatro UTI neonatais, do estado de Santa Catarina com amostra de 181 profissionais. Observou que a avaliação do grau de segurança foi de (83\%) para muito boa e aceitável já a dimensão recebeu (36\%) de respostas positivas.

Segundo Mello e Barbosa (2017), a discrepância nos estudos supracitados se deve a percepção dos profissionais de que há problemas na segurança do paciente o que culmina em uma pior avaliação da segurança do paciente, no entanto, caso o mesmo tenha percepção positiva sobre as medidas preventivas de erros a avalição será positiva. Outra justificativa seria o fato de que os dois estudos foram realizados em hospitais públicos enquanto o presente estudo foi realizado em um hospital particular de alta complexidade.

Estudo comparativo realizado por Andrade et al. (2018), em hospitais com diferentes tipos de gestões - federal, estadual e privada-observou que os fatores sobrecarga de trabalho e déficits na infraestrutura além de queixas dos próprios gestores a respeito de financiamento insuficiente para aquisição de insumos, medicamentos e equipamentos seriam possíveis justificativas para apenas o hospital privado ter 
atingido níveis de fortaleza $(>75 \%$ ) de respostas positivas. O que pode vir a justificar a diferença entre os achados na literatura e os resultados do presente estudo.

O fato das dimensões "Expectativas sobre o supervisor/ chefe" (79\%), ter sido classificada como fortalecida e o expressivo porcentual de respostas positivas atribuído a dimensão "Trabalho em equipe entre as unidades" $(73 \%)$, sugere que a equipe se sente apoiada dentro da unidade por seus supervisores além demonstrar ser um ambiente harmônico de trabalho em que os profissionais estão dispostos a ajudar os colegas de trabalho diante de adversidades (MELLO; BARBOSA, 2013).

Pesquisas que visem o estudo da cultura de segurança do paciente no setor de hemodinâmica ainda são raros na literatura. Por ser uma área que oferece riscos, tanto ao paciente, quanto ao profissional, deve ser melhorar explorada. Uma vez que, se trata de um ambiente com risco de contaminação por microrganismos, acidentes com instrumentos perfurocortantes, alta carga emocional e mental que somados caracterizam-se como um conjunto de riscos específicos do setor (NASCIMENTO, 2013).

No presente estudo foi possível observar que, embora tenha percentuais de respostas positivas superiores às encontradas em outros estudos o setor apresenta áreas com potencial de melhora. Principalmente, na necessidade de uma cultura não punitiva, que estimule as notificações para que seja possível conhecer e combater com mais eficiência as causas e consequências dos erros e eventos adversos do setor (SOUZA et. al., 2015).

\section{CONSIDERAÇÕES FINAIS}

Através desta pesquisa foi possível identificar que o setor é comporto em sua maioria por mulheres, com predomínio de indivíduos com idade entre 31 a 40 anos e profissionais médicos, enfermeiros e técnico de enfermagem. Embora apresente resultados melhores se comparado à literatura explorada o setor mostra ter potencial de melhora na maioria dos aspetos estudados uma vez que só 4 das 12 dimensão foram classificados como fortalecidas. Além disso, observou-se um baixo índice de 
notificações no setor o que contradiz a percepção dos profissionais quanto a frequência de notificações. Fato que pode ter sido influenciado diretamente pela cultura punitiva presente no setor.

Os resultados encontrados podem nortear o desenvolvimento de novas estratégias com objetivo solucionar a subnotificação do setor e do caráter punitivo as notificações que são realizadas para que assim se possam conhecer os eventos adversos de setor e evitar recidivas e ter como consequência a melhora na cultura de segurança do paciente no setor.

Sugere-se o desenvolvimento de novas pesquisas sobre segurança do paciente no setor hemodinâmico dado a sua importância e a carência na literatura de artigos que abordem tal assunto.

\section{REFERÊNCIAS}

ALVES, Kisna Yasmin Andrade; SANTOS, Viviane Euzébia Pereira; DANTAS, Cilene Nunes .A análise do conceito segurança do paciente: a visão evolucionária de Rodgers. Aquichan, Chía, v. 15, n. 4, p. 521-528, 2015.

ANDRADE, Luiz Eduardo Lima et. al., Cultura de segurança do paciente em três hospitais brasileiros com diferentes tipos de gestão. Ciênc. Saúde Colet., v.23, n.1, p. 161-172, 2018. Disponível em:< https://www.scielosp.org/article/csc/2018.v23n1/161172/pt/>. Acesso 12/08/ 2019.

BRASIL. Agência Nacional de Vigilância Sanitária. Assistência Segura: Uma Reflexão Teórica Aplicada à Prática Agência Nacional de Vigilância Sanitária. 2 ed. Brasília: ANVISA, 2017. Disponível em: $<$ http://portal.anvisa.gov.br/documents/33852/3507912/Caderno+1++Assist\%C3\%AAncia+Segura++Uma+Reflex\%C3\%A30+Te\%C3\%B3rica+Aplicada+ \%C3\%A0+Pr\%C3\%A1tica/97881798-cea0-4974-9d9b-077528ea1573> Acesso em: 24/08/ 2019. 
BRASIL. Ministério da saúde. Núcleo de segurança do paciente, Abr. 2016. Disponível em:< http://www.saude.gov.br/acoes-e-programas/programa-nacional-deseguranca-do-paciente-pnsp/nucleo-de-seguranca-do-paciente> Acesso em: 23/08/ 2019.

Brasil. Ministério da Saúde. Portaria GM/MS № 529, de 1ํ de abril de 2013.

COSTA ,Daniele Bernardi da. Cultura de segurança do paciente em serviços de enfermagem hospitalar. 2014. 98f. Dissertação (mestre em saúde)- Escola de Enfermagem de Ribeirão Preto, Universidade de São Paulo, Ribeirão Preto,2014. Disponível em: <https://www.teses.usp.br/teses/disponiveis/22/22132/tde-06022015192856/pt-br.php>. Acesso em 07/08/2019.

DIAS, Jéssica David et al. Compreensão de enfermeiros sobre segurança do paciente e erros de medicação. Rev Min Enferm., Belo Horizonte, v.18, n.4, p 866-873; out./dez. 2014.Disponível em:< https://www.reme.org.br/artigo/detalhes/969> Acesso em 07/08/2019.

DUARTE, Sabrina da Costa Machado et al. Eventos adversos e segurança na assistência de enfermagem. Rev Bras Enferm, Rio de Janeiro, v. 68, n. 1, p. 144-154, 2015.

MELLO, Janeide Freitas de,BARBOSA, Sayonara de Fátima Faria. Cultura de segurança do paciente em terapia intensiva: recomendações da enfermagem. Texto \& contexto enferm., Florianópolis,v.22, n. 4,p. 1124-1133, Out./Dez., 2013.

MELLO, Janeide Freitas; BARBOSA, Sayonara Fátima Faria. Cultura de segurança do paciente em unidade de terapia intensiva: perspectiva da equipe de enfermagem. Rev. eletrônica enferm., Goiania v. 19, 2017.

MILAGRES, L. M. Gestão de riscos para segurança do paciente: o enfermeiro e a notificação dos eventos adversos. 2015. 80f. Dissertação (mestre em Tecnologia e comunicação no cuidado em saúde e enfermagem) - Universidade Federal de Juiz de Fora, Juiz de Fora, 2015. Disponível em: 
:<http://www.ufff.br/pgenfermagem/files/2010/05/Disserta

\%С3\%A7\%C3\%A30-

Lidiane-Miranda-Milagres.pdf/>. Acesso 12/08/ 2019.

MINUZZI, Ana Paula; SALUM, Nádia Chiodelli, LOCKS; Melissa Orlandi Honório. Avaliação da cultura de segurança do paciente em terapia intensiva na perspectiva da equipe de saúde. Texto \& contexto enferm., , v.25,n.2. 2016.

NASCIMENTO, Vidal do Nascimento; CARVALHO, Yanara Pinheiro; SOUSA, Pryscila Correia de. Conhecimento dos riscos do setor de hemodinâmica: uma investigação com profissionais de saúde. In: seminário nacional de pesquisa em enfermagem,17,2013,Natal.Anais do 17 SENPE,RN:ABEn.p.1834-1836, 2013.

NEVES, Marta Isabel Cláudio. Eventos hemorrágicos no doente submetido a cateterismo cardíaco. 2017. 94f. Dissertação (mestre em Engenharia Biomédica) Instituto Superior de Engenharia de Lisboa - Escola Superior de Tecnologia da Saúde de Lisboa,Lisboa, $2017 . \quad$ Disponível em:< https://repositorio.ipl.pt/handle/10400.21/8356> Acesso em: 16/10/2019.

OLIVEIRA, Roberta Meneses et al. Estratégias para promover segurança do paciente: da identificação dos riscos às práticas baseadas em evidências. Esc Anna Nery, Rio de Janeiro, v. 18, n. 1, p. 122-129, 2014.

REIS, Claudia Tartaglia. A cultura de segurança do paciente: validação de um instrumento de mensuração para o contexto hospitalar brasileiro. 2013.217f. Tese (Doutorado em ciências na área de saúde pública) - Escola Nacional de Saúde Pública Sergio Arouca, Rio de Janeiro, 2013. Disponível em:<https://www.arca.fiocruz.br/handle/icict/14358> Acesso em: 11/08/2019.

SANTIAGO, Thaiana Helena Roma ; TURRINI, Ruth Natalia Teresa . Cultura e clima organizacional para segurança do paciente em Unidades de Terapia Intensiva. Rev. Esc. Enferm. USP., São Pauo, p. 123-130, 2015. 
SIMA N, Andréia Guerra; CUNHA, Simone Graziele Silva; BRITO, Maria José Menezes. A prática de notificação de eventos adversos em um hospital de ensino. Rev esc enferm USP, São Paulo, v. 51, 2017.

SORRA, Joann; NIEVA, Veronica. Survey on Patient Safety Culture. Rockville, MD: Agency for Healthcare Research and Quality, 2004.

SOUZA, Verusca Soares de et al. Erros e eventos adversos: a interface com a cultura de segurança dos profissionais de saúde. Cogitare enferm., Curitiba, v. 20, n. 3,p. 475482, 2015.

TOMAZONI, Andréia et al. Avaliação da cultura de segurança do paciente em terapia intensiva neonatal. Texto \& contexto enferm., Florianopolis v. 24, n. 1, p. 161-169, 2015.

WEGNER, Wiliam et al. Educação para cultura da segurança do paciente: Implicações para a formação profissional. Esc Anna Nery, Rio de Janeiro, v. 20,n.3, 2016.

Enviado: Maio, 2020.

Aprovado: Julho, 2020. 